\title{
Intencionalidad colectiva y estructura ontológica del derecho Una respuesta a Richard Ekins y Damiano Canale
}

\author{
Carlos Bernal Pulido* \\ (Traducción del inglés por Hernán Bouvier)
}

Resumen: El texto responde a las críticas realizadas por Damiano Canale y Richard Ekins en esta misma revista al artículo "Austin, Hart, Shapiro: tres variaciones alrededor del derecho como una entidad fundada en una práctica social".

Palabras clave: derecho, práctica social, grupos.

Abstract: The article answers the critics advanced by Damiano Canale and Richard Ekins in this review to the essay "Austin, Hart, Shapiro: tres variaciones alrededor del derecho como una entidad fundada en una práctica social".

Key words: law, social practice, groups.

\section{Introducción}

En "Hechos, razones y acción colectiva: reflexiones en torno a la ontología social del derecho", y "¿El derecho está basado en la acción colectiva?", Richard Ekins y Damiano Canale, respectivamente, plantean múltiples objeciones a las perspectivas que defiendo en el artículo motivo de esta discusión ("Austin, Hart, Shapiro: tres

* Macquarie Law School, (Sídney, Australia). 
variaciones alrededor del derecho como una entidad fundada en una práctica social"). El objetivo principal de tal texto fue explorar en qué medida las teorías del derecho de Austin, Hart y Shapiro proveen los fundamentos apropiados para explicar la intuición según la cual el derecho posee la estructura ontológica de una práctica social. Llamo a esta intuición tesis de la práctica social. Esta tesis sostiene que el derecho está basado en un conjunto recurrente de acciones colectivas intencionales llevadas adelante por ciertos agentes individuales, actuando juntos, en grupos, de acuerdo con, y en virtud de, sus intenciones comunes [we-intentions] y un conocimiento compartido apropiado acerca de la acción de los grupos relevantes y las intenciones comunes de sus miembros.

Ekins y Canale formulan observaciones agudas y me honran con críticas profundas respecto a mi lectura de Austin, Hart y Shapiro y a mi comprensión de la relación entre la intencionalidad colectiva, por un lado, y la estructura ontológica del derecho, por el otro. También llevan a cabo sugerencias interesantes como, por ejemplo, la que formula Ekins acerca de las raíces de la tesis de la práctica social en la obra de Tomás de Aquino; la del mismo Ekins en tono crítico acerca de la forma en que Shapiro combina la acción colectiva y la alienación; o la defensa de Canale de la concepción austiniana de la normatividad del derecho frente a las críticas de Hart. No obstante, voy a focalizarme en las objeciones más relevantes que formulan contra mis concepciones. Responderlas me ayudará a refinar mis ideas acerca de cómo el aparato conceptual de la ontología social nos puede ayudar a iluminar la naturaleza del derecho.

\section{Una lectura de los clásicos}

En "¿El derecho está basado en la acción colectiva?” Damiano Canale se ocupa de la estructura lógica de mi artículo "Austin, Hart y Shapiro". Canale interpreta su estructura de la siguiente manera. Las explicaciones de la práctica jurídica llevadas a cabo por Austin y Hart no encajan de manera completa con una explicación colectiva intencional de esa práctica. De manera tal que mientras aquellas 
explicaciones son falsas, la explicación en términos colectivos intencionales es informativa y verdadera.

Esta no es la agenda subyacente a mi análisis de las teorías de Austin, Hart y Shapiro. Mi punto de partida consiste en una afirmación modesta, a saber: que el uso del aparato conceptual de la ontología social puede iluminar nuestra comprensión de la naturaleza del derecho. Esta afirmación expresa una intuición que se encuentra tras algunas contribuciones recientes a la teoría general del derecho como la de Christopher Kutz en Complicity ${ }^{1}$, Rodrigo Sanchez Brigido en Groups, Rules and Legal Practice ${ }^{2}$, y, la más sofisticada, de Scott Shapiro en Legality ${ }^{3}$.

Lo que intenté mostrar en "Austin, Hart and Shapiro" es que tal abordaje no es completamente novedoso. Algunas de las intuiciones básicas se encontraban ya en la idea de Austin de hábito de obediencia y en la concepción de Hart sobre la práctica de aceptación de reglas sociales.

Naturalmente, soy del todo consciente que los desarrollos más relevantes en el ámbito de la ontología social, emprendidos por autores como Michael Bratman, Margaret Gilbert, Seumas Miller, John Searle y Raimo Tuomela, no estaban a disposición de Austin y Hart. No obstante, estos teóricos del derecho fueron capaces de dar cuenta de manera notable de algunas intuiciones relativas a la idea según la cual el derecho es algo que hacemos conjuntamente, actuando en grupos.

En este sentido, y esto se relaciona con la segunda objeción de Canale, ni he intentado evaluar a Austin y Hart en búsqueda de una respuesta a preguntas teóricas que preocupan a los teóricos del derecho hoy en día ${ }^{4}$, tales como el modo de explicar el derecho como una

1 Kutz, C., Complicity: Ethics and Law for a Collective Age, Cambridge, Cambridge UP, 2000.

2 Sánchez Bridigo, R. E., Groups, Rules and Legal Practice, Dordrecht: London, Springer, 2010.

3 Shapiro, S., Legality, Cambridge, Harvard University Press, 2011.

4 Canale, D., "¿El derecho está fundado en la acción colectiva? Comentario al artículo de Carlos Bernal”, Discusiones, XIV, 2014. 
actividad intencional colectiva, ni tampoco he tratado de "evaluar los problemas filosóficos que dichos autores buscaron resolver, el marco teórico utilizado para ello, y su marco contextual"5. Mi investigación de las teorías de Austin y Hart solo pretendía encontrar en la tradición positivista elaboraciones seminales de la intuición según la cual la práctica jurídica es una actividad colectiva intencional.

\section{III.La naturaleza del derecho y el rol de las razones en la naturaleza del derecho}

La primera objeción que Ekins plantea en "Hechos, razones y acción colectiva: reflexiones en torno a la ontología social del derecho" asume que defiendo una reducción de la naturaleza del derecho a hechos sociales. En contraposición, sostiene que la naturaleza del derecho no solo incluye la realidad social sino también las razones. De acuerdo con Ekins "La realidad del derecho debe encontrarse en las razones y en los hechos"'. El derecho, explica, "no consiste solo en una realidad social, dado que [...] cambia las razones que las personas tienen para actuar"7. Se sigue que toda visión acerca de la naturaleza del derecho "debe esforzarse para explicar si, y cómo, los hechos sociales dan origen a razones para la acción"

Esta objeción me da la oportunidad de señalar que no defiendo una reducción de la naturaleza del derecho a hechos, en particular, a hechos sociales. No me ocupo de tal cuestión en el texto de este volumen. En cualquier caso, soy de la idea que entender la práctica jurídica como actividad colectiva intencional no necesariamente implica estar de acuerdo con el positivismo jurídico. En contra de Shapiro, creo que esta concepción de la práctica jurídica ni excluye la posibilidad de un anti-positivismo jurídico (incluido el derecho natural) ni prueba que

5 Ibid.

6 Ekins, R., "Hechos, razones y acción colectiva: reflexiones en torno a la ontología social del derecho", Discusiones, XIV, 2014, pág. 114.

7 Ibid. 
el positivismo jurídico sea verdadero. Ciertamente, es posible usar el aparato conceptual de la ontología social para diseñar una explicación jurídica positivista sobre el derecho que reduce este a hechos sociales. Tal propuesta podría sostener que (1) el derecho puede ser reducido a la práctica jurídica; (2) toda práctica jurídica consiste en una actividad colectiva intencional, y (3) una actividad colectiva intencional de este tipo se reduce exclusivamente a los hechos, es decir, puede entenderse solo como un conjunto de agentes que llevan a cabo acciones intencionales individuales o colectivas con (y en virtud de) sus intenciones personales y comunes [I- and we-intentions] apropiadas. Esto podría denominarse la concepción subjetiva acerca de las intenciones y la naturaleza del derecho.

Sin embargo, los defensores del antipositivismo pueden sostener una concepción alternativa. Esta puede denominarse la concepción objetiva acerca de las intenciones y la naturaleza del derecho. De acuerdo con la concepción objetiva para que una actividad intencional colectiva cuente como práctica jurídica -y no solo (por ejemplo) como la actividad de una organización criminal- los funcionarios jurídicos deben tener las intenciones personales y comunes apropiadas para formular leyes que sean compatibles con las exigencias de la justicia, la moral y la corrección 9 .

La teoría de Shapiro sobre el derecho como un plan no incluye esta exigencia sino tan solo que los "funcionarios mantengan una representación de la práctica como si esta tuviera uno o varios propósitos morales"10. Clarifica luego que

sus manifestaciones no tienen por qué ser sinceras, pero en todo caso, los funcionarios, deben hacerlas. Estas representaciones pueden adoptar múltiples formas, ya sea en discursos explícitos, actos protocolares, preámbulos de la constitución,

9 Agradezco a Robert Alexy por sus observaciones sobre esta idea. Verónica Rodriguez-Blanco sostiene una visión similar en Law and Authority under the Guise of the Good, Oxford, 2014. Ekins parece tener una intuición similar cuando, citando a Agustín, hace referencia a las intenciones de una banda de asaltantes. Véase Ekins, R., op. cit., pág. 136.

${ }^{10}$ Shapiro, S., op. cit., pág. 217. 
prólogos a los códigos, decisiones judiciales, o implícitamente por medio de la ritualidad en la vestimenta o la forma de hablar, la construcción de edificios monumentales que cobijan la actividad jurídica, y el uso de iconografía moral o religiosa en escenarios jurídicos ${ }^{11}$.

Así presentado, esta exigencia es problemática. Por un lado, las bandas de ladrones pueden llevar a cabo declaraciones insinceras y esto no transforma a sus actividades colectivas intencionales en una práctica jurídica. Por otro, desde el punto de vista conceptual, nuestras intuiciones acerca del derecho sí que exigen que los funcionarios jurídicos tengan la intención sincera de actuar de un modo moralmente legítimo. Esto puede mostrarse indicando la paradoja performativa a la que se daría lugar si, por ejemplo, el preámbulo de una constitución estableciera: "Nosotros, el pueblo, sancionamos esta constitución que incluye las siguientes reglas extremadamente injustas....". Esto es evidentemente absurdo y, como explica Alexy, el absurdo se debe a una paradoja performativa generada por el conflicto entre su contenido y una presuposición pragmática, a saber: que una constitución es conceptualmente entendida como un conjunto de reglas justas, y que los funcionarios que sancionan estas reglas deben hacerlo con la intención sincera de actuar de un modo moralmente legítimo ${ }^{12}$.

Además, la afirmación de Ekins sobre el rol de las razones en la naturaleza del derecho no es de hecho incompatible con el proyecto de dar cuenta de dicha naturaleza apelando al aparato conceptual de la intencionalidad colectiva. Me referiré a esto como el proyecto de la intencionalidad jurídica. El núcleo de este proyecto consiste en la idea según la cual la realidad de las actividades jurídicas depende de la habilidad que tenemos de actuar colectivamente, es decir, como miembros de grupos o sujetos plurales.

Nosotros, actuando juntos, somos capaces de construir y sostener una práctica jurídica mediante la creación y seguimiento de las normas

11 Ibid.

12 Sobre esta paradoja performativa, véase: Alexy, R., The Argument from Injustice. A Reply to Legal Positivism, Oxford, Oxford UP, 2002, pág. 39. 
jurídicas. La creación y seguimiento de una norma jurídica exige nuestro actuar intencional, es decir, con las intenciones individuales y colectivas apropiadas para crear o seguir esa norma específica. Estas intenciones individuales y colectivas se orientan hacia la acción futura. De una manera u otra, ellas implican un compromiso prima facie del agente hacia cierto curso de acción.

Una multiplicidad de razones hacen racional para un agente mantener tal compromiso. Entre las razones de esta clase se encuentran evitar el costo de la deliberación permanente acerca de lo que debe hacerse, evitar confusiones acerca de lo que se espera que deba hacerse, evitar ser sancionado y el deseo de lograr ciertos objetivos. De este modo, las intenciones relativas a crear o seguir reglas proveen a los agentes razones prima facie para actuar de manera correspondiente ${ }^{13}$. Como consecuencia, la tarea, resaltada por Ekins, consistente en dar cuenta del modo en que el derecho crea razones para la acción, es un elemento del proyecto de la intencionalidad jurídica.

Este elemento es complejo. La complejidad deriva del hecho de que las razones expresadas mediante la participación de los agentes individuales en el proceso colectivo de crear y seguir normas son solo razones prima facie. Como ha indicado Sherwin,

a pesar de que sea racional para los agentes el comprometerse a seguir las reglas del sistema en todos (o casi todos) los casos a los cuales se aplican, no es racional para los agentes seguir las reglas cuando estas parecen exigir consecuencias incorrectas en los casos particulares ${ }^{14}$.

Por lo tanto, el proyecto de la intencionalidad jurídica colectiva debería crear un modelo capaz de explicar cuándo una razón prima facie para actuar de acuerdo con el plan es derrotable debido a la existencia de razones morales opuestas más fuertes.

${ }^{13}$ Sobre las relaciones complejas entre intenciones y acciones, véase la colección de ensayos editada por Verbeek, B., Reasons and Intentions, Aldershot, 2008.

${ }^{14}$ Sherwin, E., "Legality and Rationality: A Comment on Scott Shapiro's Legality", en Legal Theory, 19, 4, 2013, págs. 1, 6 y 7. 


\section{Actuar en grupos}

En "Austin, Hart y Shapiro" sostengo que las acciones colectivas intencionales que dan lugar y sostienen la práctica jurídica no son las acciones de un agente colectivo con una mente e intenciones propias. En algún sentido, Ekins desacuerda con esta concepción. Apoyado en el trabajo de Pettit y List sobre las actuaciones grupales, Ekins sostiene que:

uno debe considerar la posibilidad de que la ley que gobierna a las personas que forman un grupo de agentes sea, de alguna manera, producto del acto de un agente singular. De una forma más modesta, uno debería al menos considerar la forma en que las instituciones jurídicas y políticas -tribunales, ministerios y legislaturas- forman agentes grupales disciplinados para actuar conjuntamente como una persona racional natural. ${ }^{15}$

Estoy en desacuerdo con esta afirmación (que Ekins explica con más detalle en su libro The Nature of Legislative Intent ${ }^{16}$ ), según la cual, el acto de un grupo es el acto de un agente singular. List y Pettit por su parte, desde una perspectiva funcionalista, provocativamente sostienen que los agentes grupales son "entidades relativamente autónomas, agentes a título propio y con mentes propias"17 aunque explícitamente rechazan la idea de que "el actuar en grupos exija algo más que la emergencia de disposiciones coordinadas y psicológicamente inteligibles en los miembros individuales"18. De acuerdo con List y Pettit, los agentes grupales supervienen holísticamente a partir de sus miembros. Esto quiere decir que los agregados de intenciones individuales producen intenciones de grupo y que las acciones y los hechos individuales determinan las acciones y los hechos del grupo ${ }^{19}$. ¿Niega Ekins esto?

${ }^{15}$ Ekins, R., op. cit., pág. 116.

${ }^{16}$ Ekins, R., The Nature of Legislative Intent, Oxford, Oxford UP, 2012, págs. 236-43.

${ }^{17}$ Pettit P. y List, C., Group Agency, Oxford, Oxford UP 2011, pág. 77.

18 Ibid. pág. 9.

${ }^{19}$ Ibid. pág. 65. 
Ekins parece abandonar esta asunción. Por ejemplo, en su análisis de la intención legislativa, que él entiende como "el plan que la ley tal cual fue sancionada establece para la comunidad" 20 , Ekins niega que el contenido de este plan esté constituido por las intenciones entrelazadas [interlocking intentions] de los legisladores que la crearon ${ }^{21}$. Si el contenido del plan no está constituido por las intenciones entrelazadas de los legisladores, surgen al menos dos posibilidades. O bien las acciones del grupo no supervienen en las acciones de sus miembros, o bien lo que tiene lugar es un caso de la acción representativa [proxy agency], en la cual un grupo actúa por medio de ciertos agentes pero como no todos los miembros del grupo están involucrados, y a pesar de que es el grupo el que actúa, el grupo debe ser tomado como un agente autónomo e independiente con respecto a las acciones y actitudes de sus miembros individuales.

Ekins parece describir la promulgación de las leyes como un caso especial de acción representativa, esto es, como un caso en que alguien redacta y expide la ley "en nombre de la legislatura, entendida como un todo"22. En la acción representativa "una persona o subgrupo llevan a cabo algo que cuenta o constituye o es reconocido como (equivalente a) la realización de algo por otra persona o grupo" 23 . Algunos autores en el campo de la intencionalidad colectiva consideran a la acción representativa como un contraejemplo a la concepción según la cual la acción de un grupo no es más que la simple suma de las contribuciones de los miembros individuales del grupo al grupo mismo ${ }^{24}$.

No obstante, como muestra Ludwig, es posible dar cuenta de la acción representativa mediante "una explicación deflacionaria de la intencionalidad colectiva de acuerdo con la cual que el grupo de personas haga algo consiste en que sus miembros individualmente

${ }^{20}$ Ekins, R., The Nature of Legislative Intent, págs. 230-31.

${ }^{21}$ Sobre este punto Goldsworthy, J., "Legislative Intention Vindicated?" en Oxford Journal of Legal Studies, 33, 4, 2013, pág. 828.

${ }^{22}$ Ekins, R., The Nature of Legislative Intent, pág. 235.

${ }^{23}$ Ludwig, K., "Proxy Agency in Collective Action", en Noûs, 31, 1, 2013, p. 76.

${ }^{24}$ Véase, por ejemplo, Tuomela, R., The Importance of Us: A Philosophical Study of Basic Social Notions, Stanford, Stanford UP, 1995, pág. 142. 
contribuyan a llevar a cabo ese algo". ${ }^{25}$ Ludwig muestra esto en cinco pasos. Tómese "AR" como agente representante [proxy agent] , "G" como grupo que es representado, $\mathrm{y}$ " $\mathrm{A}$ " por auditorio (un individuo o grupo con el cual $\mathrm{G}$ potencialmente tiene que interactuar) ${ }^{26}$.

El primer paso es reconocer que la acción representativa implica dos acuerdos. Por un lado, de los miembros de G entre sí y con AR de que AR actuará en su nombre. Por el otro, que los miembros del auditorio $\mathrm{A}$ acuerdan que los miembros de $\mathrm{G}$ reconocen que $\mathrm{AR}$ actuará en su nombre. El segundo paso consiste en señalar que, en virtud de los acuerdos, AR posee un status funcional particular (o rol) como un agente autorizado para actuar en nombre de los miembros de $\mathrm{G}^{27}$. Una persona tiene este status funcional si es generalmente aceptado en la comunidad que ella posee la función de agente autorizado para ciertas transacciones sociales (entendidas esencialmente como acciones intencionales colectivas) ${ }^{28}$. En virtud del acuerdo entre los miembros de A y $G, A R$ es visto como un agente autorizado de los miembros de $G$ con respecto a A. Este reconocimiento habilita a AR a cumplir esta función. El tercer paso es reconocer que los miembros de $\mathrm{G}$ pueden conferir a AR su status funcional mediante el seguimiento de una regla constitutiva. Una regla constitutiva crea o constituye un tipo de comportamiento en el sentido de que la regla define el comportamiento y que este tiene lugar cuando la regla es seguida intencionalmente y no cuando tiene lugar de otra forma ${ }^{29}$. El comportamiento relevante consiste aquí en autorizar [empower] a alguien como agente representativo. Una regla constitutiva define el autorizar a alguien como agente representativo como la acción por medio de la cual los agentes de un

${ }^{25}$ Ludwig, K., "Proxy Agency in Collective Action", en Noûs, 31, 1, 2013, pág. 76.

${ }^{26}$ Lo que sigue es una adaptación de la propuesta de Ludwig en Ibíd. págs. 89-92.

${ }^{27}$ Sobre el concepto de status funcional, véase Searle, J., The Construction of Social Reality, New York, 1995, pág. 41.

${ }^{28}$ Ludwig, K., "Proxy Agency in Collective Action", pág. 77.

${ }^{29} \mathrm{Ibíd}$., pág. 83, aunque véase secciones 3-4 para algunas cualificaciones con respecto a la segunda cláusula. 
grupo autorizan a un agente a actuar en su nombre. Siguiendo esta regla, los miembros de $\mathrm{G}$ dan lugar al otorgamiento de poder a $A R$ autorizándolo como agente representativo. Esta autorización implica la aceptación por parte de $G$ de que $A R$ actuará en su nombre y de que $\mathrm{G}$ es responsable por la acción de $\mathrm{AR}$. El cuarto paso se relaciona con el concepto de acción constitutiva. Este concepto subsume ciertas acciones individuales por medio de las cuales un agente, siguiendo una o más reglas constitutivas, "hace algo que al mismo tiempo (a) contribuye a llevar a cabo un tipo de acción colectiva al ser parcialmente constitutiva de ella y (b) es constitutiva de un tipo particular de acción que constituye un componente de esa acción colectiva" ${ }^{30}$. Al expresar su acuerdo con la designación de AR como agente representativo, cada uno de los miembros de $\mathrm{G}$ realiza una acción individual que contribuye a instanciar una acción colectiva (la autorización de AR por G) y constituye un componente de ella. En el paso final AR realiza su acción como (en tanto) agente representativo en relación con A y por esa razón A y G consideran lo que AR hace como algo que expresa la actuación de los miembros de G y no sólo de AR.

El análisis de la acción representativa muestra cómo, al menos en el ejemplo que es tomado como caso-estudio en el análisis de Ludwig, no exige algo semejante a las acciones de grupo en sentido propio, como Ekins sostiene. Tampoco es necesario postular la existencia de un agente que pervive más allá de los miembros del grupo, dado que lo que el grupo lleva a cabo, como se indicó, puede ser visto en verdad como algo reducible a las intenciones y acciones de sus miembros individuales (todos ellos contribuyen efectivamente a lo que el grupo hace en tales casos). De esto se sigue que el proyecto de la intencionalidad jurídica colectiva, que sostiene que la práctica jurídica puede ser explicada en términos de las acciones individuales y colectivas de los agentes que participan en ella, no se ve socavado por el fenómeno de la acción representativa y es compatible con el ejemplo en el que la legislatura delega la tarea de expedir las leyes y de esta manera la lleva a cabo sin que haya un agente grupal autónomo sino solo individuos 
que llevan a cabo sus múltiples contribuciones a los actos oficiales del grupo por medio de sus agentes autorizados.

\section{La lectura del hábito de obediencia en Austin como práctica colectiva}

En "Austin, Hart y Shapiro" sostuve que la explicación de Austin sobre la naturaleza del derecho, tal como es presentada en The Province of Jurisprudence Determined, puede ser interpretada como una versión de la tesis de la práctica social. Los elementos centrales de su explicación pueden ser explicitados por medio de las siguientes proposiciones: toda ley constituye una orden ${ }^{31}$; una orden es la expresión de un deseo de que alguien haga o se abstenga de llevar a cabo algún acto, respaldado por la amenaza de una sanción ${ }^{32}$; toda ley positiva es establecida por un soberano o un cuerpo soberano de personas ${ }^{33} ; \mathrm{y}$ una persona o cuerpo de personas es un soberano si se satisfacen tres condiciones. Primero, esta persona o cuerpo de personas debe ser un superior común y determinado con respecto al grueso de la sociedad. Segundo, debe existir un hábito general de obediencia a él o a ellos, esto es, el grueso de la sociedad debe tener un hábito de obediencia o sumisión a él o a ellos. Y tercero, la persona o cuerpo de personas superior no debe tener un hábito de obediencia a un superior.

Dos condiciones hacen posible interpretar la teoría de Austin del derecho en cuanto orden como una versión de la tesis de la práctica social. La primera consiste en la posibilidad de analizar el hábito de obediencia como una actividad o un conjunto de acciones recurrentes. De este modo, decir que el grueso de la población debe tener un hábito de obediencia al soberano es decir que debe realizar (o abstenerse de realizar) de manera recurrente las acciones mandadas por el soberano. La segunda consiste en la posibilidad de entender el

${ }^{31}$ Austin, J., The Province of Jurisprudence Determined, Rumble, Wilfrid E., ed., Cambridge, 21, 1995.

32 Ibíd., Pág. 21.

$156 \quad{ }^{33}$ Ibíd., Pág. 165. 
hábito general de obediencia expresado en esta actividad recurrente como una actividad intencional colectiva llevada adelante por el soberano que dicta los mandatos, conjuntamente con el grueso de la población que los obedece. Existen tres elementos del concepto de actividad colectiva intencional que están claramente institucionalizados en el hábito general de obediencia. En primer lugar, dado que debe ser general, el hábito de obediencia es una actividad realizada por muchos individuos. Ellos constituyen juntos la "generalidad" o el "grueso" de los miembros de la sociedad ${ }^{34}$. En segundo lugar, estas acciones recurrentes no son llevadas a cabo por el "grueso" de la población, entendido como una clase de super-agente sino, como se indicó antes, por medio de las acciones de los individuos que pertenecen a este grueso al actuar juntos como un grupo o como un grupo de grupos. En tercer lugar, los individuos llevan a cabo estas acciones satisfaciendo intencionalmente las exigencias expresadas por los mandatos del soberano, motivados (al menos en parte) por la amenaza de sanciones. Los agentes que obedecen al soberano tienen el deseo de llevar a cabo las acciones por él ordenadas y poseen creencias sobre cómo hacerlo, las cuales dan lugar a intenciones dirigidas a clases específicas de movimientos corporales cuyo resultado es visto como comportamientos que se adecuan a lo que ha sido ordenado.

Ekins sostiene que "no existe una buena razón" para esta lectura del hábito de obediencia de Austin en términos colectivos ${ }^{35}$. En mi lectura, el concepto de un hábito general de obediencia es ambiguo. Admite al menos dos lecturas: una distributiva y otra colectiva. De acuerdo con la lectura distributiva, el hábito general de obediencia equivale a que la mayoría de los individuos de la comunidad tengan un hábito de obediencia personal e independiente con respecto a los mandatos formulados por el soberano. El auto-interés o el temor a las sanciones pueden motivar un agente a obedecer recurrentemente al soberano. Desde el punto de vista de esta interpretación, el hábito

${ }^{34}$ Ibíd., pág. 169.

${ }^{35}$ Ekins, R., "Hechos, razones y acción colectiva: reflexiones en torno a la ontología social del derecho", op. cit. 
general de obediencia es un mero agregado de las acciones personales recurrentes de obediencia de los miembros del grueso de la población que obedecen al soberano con intenciones en primera persona [I-intentions]. Esto se encuentra en contraste con la lectura de tipo colectiva. En esta, el hábito general de obediencia consiste en una acción intencional colectiva. Esto implica que los individuos que forman el grueso de la población actúan juntos de acuerdo con, y en virtud de, algún tipo de intenciones comunes [we-intentions] apropiadas, cuyo contenido es que el grueso de la población, como grupo, lleva a cabo la actividad de obedecer los mandatos formulados por el soberano por medio de las acciones apropiadas de sus miembros.

¿Cuál es la lectura correcta? Austin no aborda directamente este asunto. De acuerdo con Ekins

Austin hace que el grupo (la sociedad) surja a partir del hábito, antes que al hábito como formado y elegido por un grupo que existe independientemente del primero. Esto significa para Austin que el grupo es quienquiera tenga el hábito de obedecer a un soberano particular. Uno podría estar tentado de decir que el grupo consiste en quienes comparten un hábito, pero en verdad no hay nada compartido aquí, hay una simple superposición. Nada en la visión de Austin sugiere que los sujetos del derecho actúen conjuntamente o tengan una relación entre ellos más allá de ser súbditos del mismo soberano, la cual es una relación entre soberano y sujeto ${ }^{36}$.

Este pasaje muestra una diferencia clave entre la interpretación de Ekins y la mía acerca de la sociedad política austiniana. En la forma en que entiende las intenciones legislativas el análisis de Ekins parte de asumir que un grupo, como un individuo, pre-existe a la práctica. En su interpretación de Austin, el grupo pre-existente es la sociedad, cuyos miembros son los sujetos del derecho. Sin embargo, es un grupo "sobre el que se actúa" 37 . Esto significa que la sociedad

36 Íbid., pág. 120.

${ }^{37}$ Ibid. 
no es "un grupo con propósito, capaz de acción colectiva" 38 . Esto significaría que solo sería plausible la lectura individualista del hábito de obediencia. Existen efectivamente en The Province of Jurisprudence múltiples pasajes que apoyan esta lectura.

Sin embargo, la lectura del hábito de obediencia en términos colectivos no puede descartarse. Algunas observaciones de Austin, y lo que puede extraerse de ellas, también sustentan una concepción colectivista. Esto se ve, por ejemplo, en la siguiente explicación sobre cómo una sociedad natural - que vive en una condición salvaje - se transforma en una sociedad política, esto es, una sociedad en la cual existe derecho porque sus miembros obedecen al soberano:

\begin{abstract}
Mientras la sociedad en cuestión vive en condición salvaje [...] la generalidad o grueso de sus miembros no se encuentra en un hábito de obediencia a uno y el mismo superior. Para el propósito de atacar un enemigo externo, o para el propósito de repeler un ataque realizado por un enemigo externo, la generalidad o grueso de sus miembros, capaces de llevar armas, se someten a un líder o un cuerpo de líderes ${ }^{39}$.
\end{abstract}

Este pasaje revela algo importante acerca de la naturaleza del hábito de obediencia ¿Cómo resulta posible que una sociedad, esto es, un número de individuos, adquiera el hábito de obediencia a un soberano? Un análisis de lo que Austin sostiene acerca de esta sociedad que vive en una condición salvaje revela algunos elementos esenciales para la adquisición del hábito. Primero, la adquisición del hábito depende de acciones de los miembros de la población. A pesar de que, con respecto al soberano, la sociedad política se encuentra en una posición de sujeción, el adquirir esta posición requiere la realización de ciertas acciones por parte de los miembros de la sociedad. En contra de lo que sostiene Ekins, no es que se actúe sobre la sociedad. Sus miembros "se someten" ["submit"] (como dice Austin) al soberano. Son ellos quienes realizan la acción de someterse [submitting]. En

${ }^{38}$ Ibid.

${ }^{39}$ Austin, J., op. cit., pág. 216. 
segundo lugar, la acción es intencional o, como dice Austin, se lleva a cabo con un propósito. En el pasaje citado Austin está claramente pensando en un propósito dirigido a una acción colectiva intencional de "atacar un enemigo externo, o...repeler el ataque de un enemigo externo". Aquí el enemigo externo es claramente concebido como un grupo y el ataque o rechazo es claramente concebido como una acción colectiva, y esta precisamente con ese propósito compartido, como una acción colectiva de los miembros del grupo con la intención colectiva de que deberían atacar o repeler al enemigo. No es como si cada uno de ellos intentase por sí mismo atacar o repeler al enemigo, sino en cambio que cada quien debe tomar parte en la realización colectiva. Por lo tanto, aunque Austin no disponía del andamiaje para hacer todas las distinciones relevantes, estaba pensando, de hecho, en la sumisión a una autoridad soberana como un medio para un fin adoptado por el grupo para llevar a cabo acciones concebidas como colectivas intencionales.

Esta explicación puede ser también utilizada para responder a la crítica de Canale de acuerdo con la cual "la noción del derecho que tiene Austin es esencialmente no cooperativa" 40 . Canale llega a esta conclusión a partir del modo en el que Austin estructura la relación de sujeción entre el soberano y los miembros de la sociedad. De acuerdo con Canale, en virtud de esta estructura, "las sociedades políticas no son grupos sociales en un sentido naturalista: no son el resultado de lo que la gente hace conjuntamente" 41 . En cambio, sostiene, ellas son "artefactos sociales" 42 .

Aparte de la dificultad a la que da lugar esta afirmación -dado que cabe preguntarse en qué sentido una sociedad política puede ser un artefacto- la concepción de Canale soslaya que la sumisión al soberano podría ser interpretada como una acción colectiva con un propósito que los miembros de la sociedad llevan adelante involucrándose en lo que Austin llama "hábito de obediencia". El soberano también

${ }^{40}$ Canale, D., op. cit., pág. 80.

41 Íbid, pág. 81.

42 Ibid. 
está involucrado en esta actividad intencional. Esta característica es incluso reconocida por Canale cuando afirma que existe un "acuerdo social implícito en la fundación del derecho"43.

\section{Sobre el punto de vista interno en Hart}

En The Concept of Law, Hart sostiene que el derecho está basado en una práctica social normativa consistente en la aceptación de reglas sociales. De acuerdo con el autor, la aceptación de una regla social implica la existencia de un hábito y tres aspectos ulteriores: que la desviación de lo previsto por la regla da lugar a críticas e imposición de sanciones; que esa crítica a la desviación y la imposición de sanciones es vista como legítima, justificada o hecha por una buena razón; y el así llamado punto de vista interno, esto es, que los agentes "deben considerar al comportamiento en cuestión como un standard general a ser seguido por el grupo como un todo" 44 . El punto de vista interno es la actitud de aceptar una regla. En el Postcript a The Concept of Law, Hart definió la actitud de aceptación de la regla de la siguiente manera: "[La aceptación] consiste en la disposición permanente de los individuos a adoptar tales patrones de conducta al mismo tiempo como guías para su conducta futura y como estándares de crítica que podrían legitimar reclamos y formas variadas de presión" 45 .

Shapiro ha sugerido que esta disposición constante debería ser interpretada como una disposición que implica una intención de actuar de acuerdo a la regla, de criticar a otros por no adecuarse a ella, y no criticar a quienes critican la desviación ${ }^{46}$. Estoy en desacuerdo con Shapiro porque su interpretación atribuye a los agentes un excesivo conocimiento auto-consciente de las reglas. Parece posible hablar de agentes que aceptan reglas sin haberse formado intenciones de seguir tales reglas. Existen numerosas reglas que rigen la vida social

${ }^{43}$ Ibid.

${ }^{44}$ Hart, H. L. A., The Concept of Law, Oxford, 1994, págs. 55-66.

${ }^{45}$ Hart, H. L. A., op. cit., pág. 225.

${ }^{46}$ Shapiro, S., "What is the Internal Point of View?", en Fordham Law Review 75, 2006, pág. 1163. 
a las que nos adecuamos, y que son el resultado de presiones sociales de variado tipo que equivalen a una forma de entrenamiento y que, sin embargo, jamás hemos articulado y presentado de manera explícita y que tampoco han sido articuladas o presentadas para nosotros de tal manera. Estas son parte de lo que John Searle ha llamado la intencionalidad de trasfondo [background of intentionality] ${ }^{47}$. Entendido de esta manera, el punto de vista interno en Hart llevaría apropiadamente a la idea según la cual la aceptación de reglas implica la existencia de una disposición a adecuarse a ellas como parte de (nuestra) intencionalidad de trasfondo, pero no implicaría un compromiso explícito (una intención) de adecuar el comportamiento al contenido de la regla.

Ekins no está de acuerdo con mi interpretación del punto de vista interno. Sostiene que esta interpretación "hace desaparecer la intelectualización" 48 . La razón del desacuerdo es que el concepto de intención de Ekins es más bien estrecho. De acuerdo con su postura "Uno puede tener una intención condicional y actuar de conformidad sin mucha auto-conciencia" 49 . Sobre esta base, interpreta el punto de vista interno como una "intención condicional" 50 . Distingue tal concepción de una que me atribuye, en la cual la educación y el entrenamiento social respecto a las reglas "condicionan a las personas a responder sin pensarlo a cierto estímulo" 51 .

Esta concepción interpretaría la disposición estándar de Hart como una "'adopción no reflexiva de normas particulares y proposiciones" 52 . Ekins sostiene que una concepción tal no solo es incompatible con la teoría de Hart (aunque no justifica por qué) sino que también es incorrecta porque "no explica cómo es que el agente considera a la regla una razón" ${ }^{53}$.

${ }^{47}$ Searle, J., Intentionality, Cambridge, 1983, capítulo 5.

${ }^{48}$ Ekins, R., "Hechos, razones y acción colectiva: reflexiones en torno a la ontología social del derecho", op. cit., pág. 125.

${ }^{49}$ Ibid.

${ }^{50}$ Ibid.

${ }^{51} \mathrm{Ibid}$.

${ }^{52}$ Ibid. 
No refrendo una interpretación del punto de vista interno en Hart como una "adopción irreflexiva" de normas como resultado de la respuesta irreflexiva de las personas frente a los estímulos. Mi idea es que la disposición constante a la que alude Hart consiste en una capacidad e inclinación mental pre-intencional -en el sentido de Searle ${ }^{54}$, de trasfondo de prácticas y asunciones pre-intencionales- de los funcionarios jurídicos que consideran a las reglas tanto como guías de su propia conducta futura así como estándares de crítica de las conductas de otros individuos. Esta concepción es consistente con una comprensión más amplia de las intenciones ${ }^{55}$, la cual posee un cierto matiz bratmaniano, al exigir un "compromiso" para actuar. Un funcionario jurídico tendrá la intención de considerar una regla jurídica como una guía de conducta solo si está comprometido a actuar de acuerdo con ella y a usarla para evaluar la conducta de otros individuos. Sería implausible sostener que la práctica jurídica está constituida por la aceptación de reglas sobre las cuales los funcionarios tienen una intención en este sentido, y que esto se refiere a todas las reglas jurídicas posibles.

Ekins interpreta la disposición continua de la que habla Hart como una intención condicional. Como se sostuvo, la posición de Ekins sobre la intención es más estricta que la mía. Sin embargo, no explicita qué clase de concepción sostiene. Su crítica plantea la compleja pregunta sobre qué clase de actitud se exigiría en su concepción para sostener que los funcionarios han aceptado una regla. Es más, ¿poseen los funcionarios jurídicos la misma actitud (una intención condicional) hacia todas las reglas del sistema jurídico y es una condición para la existencia del sistema que esto sea así? Me parece que el único modo en que Ekins puede construir el tipo de actitud que tiene en mente de manera tal de dar sentido a los hechos efectivos que subyacen a la práctica jurídica es en un modo muy débil, esto es, bastante similar a la inclinación pre-intencional presentada por mí más arriba. Si esto es

${ }^{54}$ Searle, J., The Rediscovery of the Mind, Cambridge, Mass., 1992, pág. 189.

${ }^{55}$ Sobre las diferentes concepciones de "intención", véase: Setiya, K., "Intention", en Stanford Encyclopedia of Philosophy, 2014. Disponible on-line en http://plato.stanford.edu/entries/intention/ (26.05.2014). 
así, entonces el punto es si está aquí en juego algo más que una mera diferencia verbal.

\section{La lectura individual del concepto de aceptación de regla en Hart}

Ekins no está de acuerdo con mi concepción según la cual el concepto de aceptación de regla en Hart admite una lectura distributiva o colectiva. De acuerdo con la lectura distributiva, cada uno de los miembros de la sociedad obedece las reglas, critica la desviación, considera esta crítica legítima y tiene una disposición individual a tomar ciertas reglas sociales como patrones de comportamiento para sí y la sociedad en su conjunto. Bajo esta interpretación, la aceptación de reglas consistiría en la combinación de dos elementos: un elemento externo, esto es, un conjunto de acciones recurrentes agregativas de conformidad a ciertas reglas sociales en que se critica la desviación y se considera tal crítica como legítima, y un elemento interno, es decir, un conjunto de disposiciones individuales agregativas de conformarse a ellas y de criticar la desviación. El elemento interno constituye una actitud en primera persona [I-attitude] y no una actitud común [we-attitude]. En la lectura distributiva, la aceptación de una regla consiste en la formación de una disposición individual a guiar el comportamiento de acuerdo con la regla. Por el contrario, en la lectura colectiva, la aceptación de una regla puede ser entendida en el sentido de que en un grupo se forman las intenciones comunes condicionales apropiadas de actuar de acuerdo con las reglas en las circunstancias apropiadas ${ }^{56}$.

A pesar de que Hart no provea clarificaciones ulteriores sobre este aspecto, Ekins no está de acuerdo con la posibilidad de una lectura distributiva. Su argumento central es que Hart se ocupa de reglas sociales y

${ }^{56}$ Canale en el texto de este volumen parece estar de acuerdo conmigo acerca de la lectura distributiva de la aceptación de reglas en Hart. Véase: Canale, D., op. cit., pág. 87. 
que las reglas sociales "rigen un grupo" 57 . Por lo tanto, "las reglas son las reglas de un grupo en cuanto tal (en vez de conjunto de individuos)" 58 .

Estoy de acuerdo con Ekins en que, aunque Hart no aclare si la actitud de las personas que aceptan las reglas sociales -ciudadanos o funcionarios jurídicos- consiste en una intención individual o colectiva (intenciones en primera persona o intenciones comunes, en el vocabulario de la ontología social) la lectura colectiva de la estructura de la aceptación de reglas sociales encuentra mayor apoyo en el texto de Hart que la lectura distributiva. Sin embargo, rechazo lo que el argumento de Ekins podría abrigar, a saber: que la aceptación de reglas sociales es necesariamente una actividad colectiva intencional.

Con respecto a este asunto específico, Ekins pregunta de manera retórica (en especial con respecto a la regla de reconocimiento):

¿Tendría sentido para una persona aceptar esta regla [la regla de reconocimiento] con independencia de la intención de otros? Sería sorprendente, porque el punto de la regla es proveer una regla para el grupo, de modo que es muy importante que todos (o la mayoría) acepten la misma regla.

Existe una distinción entre intentar contribuir a una acción de un grupo con una intención en primera persona e intentarlo con una actitud común. Por lo tanto existen dos lecturas con respecto a las intenciones cuyos contenidos están dirigidos a la participación de los individuos en acciones de grupos. Siguiendo la interpretación de Hart que hace Ekins, deberíamos considerar que la expresión "aceptar la regla social R" denota una intención condicional de adecuarse en circunstancias apropiadas a su contenido. Supongamos que aceptar la regla exige que los demás también posean una intención condicional similar. La pregunta es si esta naturaleza condicional transforma a esta intención ipso facto en una intención común.

57 "Ekins, R., "Hechos, razones y acción colectiva: reflexiones en torno a la ontología social del derecho", op. cit.

${ }^{58} \mathrm{Ibid}$, pág. 128. 
Mientras que Ekins daría una respuesta afirmativa a esta pregunta, yo le daría una respuesta negativa. Pueden existir acciones de grupo en las cuales cada individuo contribuye a la acción del mismo con una intención en primera persona. Un ejemplo es el siguiente: supóngase que quiero que todo el mundo abandone un teatro, incluido yo mismo. Formo la intención de realizarlo eligiendo pararme y gritando "fuego" en voz alta. Supóngase que, de hecho, todo el mundo deja su asiento y como resultado todos abandonamos el teatro. Todos teníamos la intención de abandonar el teatro, y así lo hicimos como resultado, pero no compartíamos la intención de hacerlo, y no lo hicimos intencionalmente en forma conjunta. Cada uno de nosotros lo hizo con una intención en primera persona y no con una intención común ${ }^{59}$.

Una pregunta relevante es si aceptar o seguir reglas sociales necesariamente implica para los miembros del grupo que acepten o sigan la regla el realizar sus contribuciones con intenciones comunes. Esto depende del tipo específico de regla social que podamos considerar. El seguimiento de ciertos tipos de reglas sociales no implica ipso facto que los miembros lo lleven a cabo con una intención común y se involucren en un comportamiento intencional colectivo. Un ejemplo es la regla de mantener cierta distancia con los interlocutores en una conversación. Esta es una regla social neutral con respecto a si la acción colectiva que rige es conjuntamente intencional o no. Es perfectamente posible para dos individuos aceptar la regla (intentar condicionalmente seguirla en las circunstancias apropiadas) solo con intenciones en primera persona.

En este escenario, un enunciado que describe la aceptación de esta regla: "ellos aceptan conservar la distancia apropiada con sus interlocutores o compañeros de conversación" debería ser leída como "cada uno de ellos tiene la intención de mantener él con todo interlocutor o compañeros de conversación una distancia apropiada". Sin embargo, es también posible que alguien que acepta la regla posea una intención

${ }^{59}$ Agradezco a Kirk Ludwig por el ejemplo, y los ejemplos que siguen sobre el teatro y el saludo, además del valioso intercambio sobre la diferencia entre contribuir a un grupo con una intención en primera persona y una intención común. 
común de que él y otros hagan eso, o es posible que ambos tengan intenciones comunes, pero no es necesario.

En contraste, existen también reglas sociales que rigen esencialmente acciones de tipo intencional colectivo. Con respecto a estas es imposible para un individuo intentar seguirlas sin una intención colectiva de que aquellos con quien actúa asimismo intenten seguirla con una intención común. Un ejemplo es la regla social que especifica que cuando saludamos a otra persona quien extiende la mano derecha debería tomar la mano derecha de la otra persona y mover con el otro la mano hacia abajo y arriba. Este tipo de acción es esencialmente colectiva. Por tanto, la regla social que rige esta acción no puede ser correctamente seguida sin una intención común.

Ahora bien, en lo que respecta a Hart y Ekins el enunciado "nosotros aceptamos una regla social" o "nosotros aceptamos la regla de reconocimiento" es susceptible de ambas lecturas, esto es, una lectura distributiva en la cual los miembros del grupo aceptan la regla con intenciones de primera persona, y una lectura colectiva en la cual los miembros aceptan la regla con intenciones comunes. Hart no desarrolló este aspecto. Y la observación de Ekins de que no tiene sentido para alguien aceptar la regla de reconocimiento "con independencia de las intenciones de los otros" conduce en este punto a confusión ${ }^{60}$. Como un miembro del grupo que acepta la regla de reconocimiento, puedo tener la intención de aceptar la regla con otros miembros del grupo aunque no tenga la intención de que todos nosotros lo hagamos con la intención común de hacerlo conjuntamente [though I do not intend that we all we-intend that we do it together]. Puedo tener tan solo una intención en primera persona. Una lectura colectiva de la actitud implícita en la aceptación de reglas sociales no exigiría solo para los agentes relevantes el reconocer una regla teniendo en cuenta la intención de los otros. Lo que es necesario para los agentes -funcionarios jurídicos y ciudadanos, dependiendo del enfoque- es tener una intención común, esto es, intentar conjuntamente aceptar la regla y

${ }^{60}$ Ekins, R., "Hechos, razones y acción colectiva: reflexiones en torno a la ontología social del derecho", op. cit. 
aceptarlaintencionalmente de manera conjunta. Que el concepto de Hart de aceptación de reglas exija esta clase de actitud colectiva es materia pendiente de prueba.

\section{Sobre la relación entre prácticas sociales y reglas sociales}

Canale indica una cuestión muy relevante con respecto a la relación entre prácticas sociales y reglas sociales en la teoría de Hart. Según él "una práctica social no da lugar a reglas sociales"61. Sostiene que "El concepto de regla social simplemente explica lo que una práctica social en verdad es: por qué los miembros de un grupo se comportan de cierta manera y qué acciones se necesitan para ser miembro del grupo, es decir, un participante de la práctica."62. En este sentido, Canale acuerda con Shapiro cuando luego sostiene que Hart comete un error de categoría dado que "las reglas y las prácticas pertenecen a categorías metafísicas diferentes" 63 . Mientras las reglas son objetos abstractos, objetos del pensamiento, y no entidades que existen dentro del espacio y el tiempo, las prácticas son eventos concretos: tienen lugar dentro del mundo natural e interactúan causalmente con otros eventos físicos.

En "Austin, Hart y Shapiro", concuerdo con Shapiro en que las reglas y las prácticas pertenecen a diferentes categorías metafísicas y en que las reglas sociales son objetos abstractos mientras que las prácticas sociales son eventos concretos. Las reglas sociales son objetos de pensamiento que pueden comúnmente ser reconstruidas con la forma lógica condicional: "Si las circunstancias C tienen lugar, entonces la acción A es exigida". Las prácticas sociales son conjuntos de acciones colectivas recurrentes. Y las acciones son eventos. Por esta razón, las prácticas sociales son conjuntos de eventos y los eventos son particulares.

${ }^{61}$ Canale, D., "¿El derecho está basado en la acción colectiva?"

${ }^{62} \mathrm{Ibid}$., pág. 88.

${ }^{63}$ Shapiro, S., Legality, pág. 103. 
No obstante, también sugerí una interpretación alternativa del enfoque de Hart, de acuerdo con el cual en su teoría las reglas sociales no se reducen a prácticas sociales sino que se basan en ellas: lo que Hart, de hecho, sostiene es que una práctica social que consiste en la aceptación de la regla de reconocimiento es el fundamento [foundation] de un sistema jurídico, es decir, que la validez de todas las reglas jurídicas está basada en la práctica oficial de aceptar la regla de reconocimiento última. Esto significa que Hart no comete ninguna clase de error categorial. No sostiene que los objetos abstractos (reglas) sean reductibles a eventos concretos (práctica social) sino solo que estos objetos abstractos son válidos (para una comunidad) porque existe una práctica (en esa comunidad), esto es, un conjunto de acciones concretas intencionales de los funcionarios de aceptar la regla de reconocimiento, y de tomar esos objetos abstractos (reglas jurídicas producidas de acuerdo a la regla de reconocimiento) como estándar de la conducta futura de la sociedad en su conjunto.

Canale parece estar en desacuerdo con esta interpretación de Hart. Lo que sostengo es que el enfoque de Hart sobre la aceptación de reglas no explica si las prácticas sociales pueden fundamentar reglas sociales. Ciertamente, en la concepción de Hart, aceptar la regla de reconocimiento implica que los funcionarios jurídicos estén dispuestos a tomar tal regla como una premisa en el razonamiento práctico. Sin embargo, no implica que deban tomarla como una premisa en el razonamiento práctico ni que deban estar dispuestos a hacerlo. La disposición de los funcionarios jurídicos a tomar la regla de reconocimiento como una premisa en el razonamiento práctico ni los obliga a hacerlo ni a continuar con la disposición a hacerlo. Siguiendo a $\mathrm{Toh}^{64}$ Canale entiende que el problema de si las prácticas sociales pueden fundamentar reglas sociales resulta irrelevante en la teoría de $\mathrm{Hart}^{65}$. Sin embargo, esto transformaría su teoría en una demasiado liviana y sería inconsistente consu interés de elaborar la naturaleza de la obligación jurídica (que

${ }^{64}$ Toh, K., "The Predication Thesis and a New Problem about Persistent Fundamental Legal Controversies", en Utilitas 22, 3, 2010, pág. 333.

${ }^{65}$ Canale, D., op. cit. 
es diferente del simple verse obligado y de la obligación moral) y de las propiedades que dan lugar a la validez de las reglas jurídicas.

\section{Acerca de la teoría de Shapiro sobre el derecho como plan}

Ekins y Canale realizan múltiples objeciones contra la teoría de Shapiro del derecho como plan. Por un lado, Ekins critica la propuesta de Shapiro según la cual compartir un plan funcionaría como una explicación de la acción colectiva y también el modo en el cual Shapiro entiende la relación entre la acción colectiva y la alienación. En cambio, Ekins sugiere que la realidad social del derecho debería ser entendida sobre la base de un análisis de las "buenas razones" y elecciones "bien formadas" de la gente cuyas acciones constituyen la práctica jurídica ${ }^{66}$. No obstante, Ekins no se explaya con respecto a este punto. Por otro lado, Canale realiza comentarios muy agudos acerca de la posibilidad de interpretar la teoría de Shapiro del derecho como plan apelando a una lectura distributiva y el rol que los procedimientos deberían jugar en tal enfoque. ${ }^{67}$ Canale, además, adhiere a la objeción de Celano de acuerdo con la cual los planes no pueden ser usados para la planificación social masiva ${ }^{68}$.

No me es posible comentar aquí todas estas cuestiones. Como indiqué en "Austin, Hart, Shapiro" la teoría del derecho como plan es, sin duda alguna, la explicación más desarrollada del derecho como práctica social. Sin embargo, no adhiero a la teoría de Shapiro en su totalidad. No es este el lugar para explicitar mis desacuerdos con Shapiro. No obstante, en contra de Canale y Celano, sí pienso que el aparato conceptual de la intencionalidad colectiva puede ser útil para dar cuenta de algunas características esenciales de actividades sociales masivas tales como la práctica jurídica. Explicaciones del tipo al que se hizo referencia más arriba (sobre Ludwig y la acción

${ }^{66}$ Ekins, R., "Hechos, razones y acción colectiva: reflexiones en torno a la ontología social del derecho", op. cit.

${ }^{67}$ Canale, D., op. cit.

${ }^{68}$ Ibid. 
representativa) dan lugar a la idea de que los poderes sociales y jurídicos y las reglas que confieren poderes pueden ser explicados en términos de los conceptos fundamentales de la ontología social. Los poderes (potestades) y las reglas que confieren poderes son la médula ósea de las actividades masivas. Naturalmente, el modo en el cual una explicación básica, como la de la agencia representativa, debería ser expuesta para así explicar los poderes consagrados en la constitución, cómo un enfoque de este tipo puede hacerse compatible con la naturaleza de otras normas y cómo entender la normatividad del derecho dentro de este marco resulta una empresa que todavía debe ser llevada a cabo. Esta tarea se encuentra en el horizonte del proyecto de la intencionalidad jurídica colectiva.

\section{Bibliografía}

Alexy, R., The Agument from Injustice. A Reply to Legal Positivism, Oxford, Oxford UP, 2002.

Austin, J., The Province of Jurisprudence Determined, Cambridge, Cambridge UP, 1995.

Canale, D., "¿El derecho está fundado en la acción colectiva? Comentario al artículo de Carlos Bernal", Discusiones, XIV, 2014, pág. 73-12.

Ekins, R., The Nature of Legislative Intent, Oxford, Oxford UP, 2012.

Ekins, R., "Hechos, razones y acción colectiva: reflexiones en torno a la ontología social del derecho", Discusiones, XIV, 2014, pág. 113-144.

Goldsworthy, J., "Legislative Intention Vindicated?", Oxford Journal of Legal Studies, 33, 4, 2013

Hart, H.L.A., The Concept of Law, Oxford, Clarendon Press, 1994 [1961].

Kutz, C., Complicity: Ethics and Law for a Collective Age, Cambridge, Cambridge UP, 2000.

List, C. y Pettit, P., Group Agency, Oxford, Oxford UP, 2011.

Ludwig, K., "Proxy Agency in Collective Action", Nô̂s, 31, 1, 2013 
Sánchez Brigido, R.E., Groups, Rules and Legal Practice, Dordrecht; London, Springer, 2010.

Searle, J.R., Intentionality. An essay in the philosophy of mind, Cambridge, Cambridge University Press, 1983.

Searle, J. R., The rediscovery of the mind, Cambridge, Mass. ; London, MIT Press, 1992.

Searle, J. R., The Construction of Social Reality, New York, Free Press, 1995.

Shapiro, S., "What is the Internal Point of View?", Fordham Law Review, 75, 2006, pág. 1157-1170.

Shapiro, S., Legality, Cambridge, Harvard University Press, 2011.

Sherwin, E., "Legality and Rationality: A Comment on Scott Shapiro's Legality", Legal Theory, 19, 4, 2013

Toh, K., "The Predication Thesis and a New Problem about Persistent Fundamental Legal Controversies", Utilitas, 22, 2010

Toumela, R., The Importance of Us: A Philosophical Study of Basic Social Notions, Stanford, Stanford UP, 1995." 